\title{
Dark matter inner slope and concentration in galaxies: from the Fornax dwarf to M87
}

\author{
G. A. Mamon ${ }^{1}$, J. Chevalier ${ }^{1}$, A. J. Romanowsky ${ }^{2,3}$ and R. Wojtak ${ }^{4}$ \\ ${ }^{1}$ Institut d'Astrophysique de Paris, UMR 7095 (CNRS \& UPMC), 98 bis Bd Arago, F-75014 \\ Paris, France, email: gam@iap.fr, jill.chevalier@gmail.com \\ ${ }^{2}$ Dept. of Physics and Astronomy, San Josè State University, San Josè, CA 95192, USA \\ ${ }^{3}$ Univ. of California Observatories, Santa Cruz, CA 95064, USA, email: romanow@ucolick.org \\ ${ }^{4}$ Dark Cosmology Centre, DK-2100 Copenhagen, Denmark, email: wojtak@stanford.edu
}

\begin{abstract}
We apply two new state-of-the-art methods that model the distribution of observed tracers in projected phase space to lift the mass / velocity anisotropy (VA) degeneracy and deduce constraints on the mass profiles of galaxies, as well as their VA. We first show how a distribution function based method applied to the satellite kinematics of otherwise isolated SDSS galaxies shows convincing observational evidence of age matching: red galaxies have more concentrated dark matter (DM) halos than blue galaxies of the same stellar or halo mass. Then, applying the MAMPOSSt technique to M87 (traced by its red and blue globular clusters) we find that very cuspy DM is favored, unless we release priors on DM concentration or stellar mass (leading to unconstrained slope). For the Fornax dwarf spheroidal (traced by its metal-rich and metal-poor stars), the inner DM slope is unconstrained, with weak evidence for a core if the stellar mass is fixed. This highlights how priors are crucial for DM modeling. Finally, we find that blue GCs around M87 and metal-rich stars in Fornax have tangential outer VA.
\end{abstract}

Keywords. galaxies: elliptical and lenticular, $\mathrm{cD}$ - dark matter

\section{Introduction}

While DM cusps (with slopes steeper than $\gamma=-0.9$ for radii $r>r_{-2} / 100$, i.e. $r>$ $r_{\text {vir }} / 1000$ ) are predicted in DM-only cosmological $N$-body simulations (Navarro et al. 2004), it is not clear how the astrophysics of baryons will affect the slope $\gamma$ of the inner DM density profile. Pontzen \& Governato (2012) have shown that intermittent feedback can transform the DM cusps into homogeneous cores, while hydrodynamical cosmological $N$-body simulations by Di Cintio et al. (2014) lead to the view that $\gamma$ is a function of the stellar to halo mass ratio, where $L^{*}$ ellipticals and dwarf spheroidals (dSphs) are expected to be cuspy while galaxies of intermediate mass should have DM cores.

Mass modeling of dSphs (using internal motions as the only possible method available, and now with over 1000 and 200 member velocities available for Sculptor and Fornax, respectively) leads to a variety of results. For Sculptor, Walker \& Peñarrubia (2011) used the Wolf et al. (2010) pinch (insensitivity of the mass profile to the a priori unknown velocity anisotropy [VA] at some "pinch" radius) on metal-rich ( $Z$-rich) vs. metal-poor $(Z$-poor) stars to deduce a shallow DM slope for Sculptor. However, this result has been disputed by Breddels et al. (2013) and Richardson \& Fairbairn (2014), who both found (using orbit modeling and dispersion-kurtosis Jeans modeling, respectively) that both cores and cusps were allowed for the DM. While Jardel \& Gebhardt (2012), using orbit modeling, deduced cored DM for the Fornax dSph, Amorisco et al. (2013) (using the Wolf pinch) found that while a DM core is the natural outcome for Fornax, (cuspy) NFW DM is possible if its concentration $\left(r_{\mathrm{vir}} / r_{-2}\right)$ is low. 
Similarly, two recent studies of the M87 giant elliptical (in the center of the Virgo cluster), traced by 275 to 375 globular clusters (GCs), lead to discrepant results: Murphy et al. (2011) (who also considered stars) deduced cored DM (with orbit modeling), while Agnello et al. (2014) found cuspy DM (with Jeans modeling).

\section{Methods}

There are two classes of methods to handle kinematic data of spherical systems (see Chapter 5 of the review of Courteau et al. 2014): 1) matching the line-of-sight (LOS) velocity moments predicted by the Jeans equation with those measured in bins of projected radii; 2) fitting the full distribution of observed tracers in projected phase space (PPS), using 6D distribution functions (DFs) expressed in energy and angular momentum, $f(E, J)$ (i.e. orbit modeling). Each has its weaknesses: The results with moments depend on the choice of radial bins (see Richardson \& Fairbairn 2013) and do not use the full PPS information. Orbit modeling currently cannot continuously update parameters of the mass profile.

Wojtak et al. (2009) developed a PPS method where $f(E, J)$ is separable, taken from Wojtak et al. (2008), who derived the $J$ term of the DF from first principles and checked the separable form of the DF on cluster-mass $\Lambda$ CDM halos. While this method is ideal for clusters of galaxies, where dissipational effects are thought to be unimportant, it is not clear how the separability of the DF will survive the effects of dissipation expected in galaxies. Unfortunately, this method is slow, as it involves a triple integral to express the distribution of tracers in PPS in terms of the DF (Dejonghe \& Merritt 1992).

Mamon, Biviano, \& Boué (2013) developed a very rapid alternative, called MAMPOSSt, which is as accurate. MAMPOSSt assumes a form for the 3D velocity distribution of tracers (here, Gaussian), which allows one to predict the distribution of tracers in PPS as a single integral of the LOS velocity distribution function.

\section{Age matching from galaxy mass modeling}

Analyzing, with the DF method of Wojtak et al. (2009), the kinematics of isolated SDSS galaxies, traced by their satellites (stacked in 9 bins of host galaxy stellar mass and color), Wojtak \& Mamon (2013) discovered that the DM halos of red galaxies are more concentrated ( $2 \sigma$ level) than those of blue galaxies of the same stellar or halo mass. This is a stark observational confirmation of age matching (Hearin \& Watson 2013): halos that assemble earlier (more concentrated) lead to galaxies with older stellar populations (redder). Their trends of halo concentration vs. mass for red and blue galaxies are well matched by predictions from age matching theory (Watson, private communication).

\section{M87}

We trace the DM profile of M87 using GCs. We use the SLUGGS (Strader et al. 2011) database of GCs, removing all objects classified as UCDs, or transition objects (i.e. only keeping objects with half-mass radii lower than $5.25 \mathrm{pc}$, following Strader et al.), and also removing all velocities measured before 2008 (which we suspect may contain important systematic errors). This leaves us with 143 red and 322 blue GCs out to $200 \mathrm{kpc}$. Since the spectroscopic sample is incomplete, we adopt the number density profiles based on photometric samples (Table 11 of Strader et al.).

We ran MAMPOSSt for several models. Our standard model has a generalized NFW DM profile (see Sect. 5) with the concentration-mass $(c-M)$ relation found in $\Lambda$ CDM 

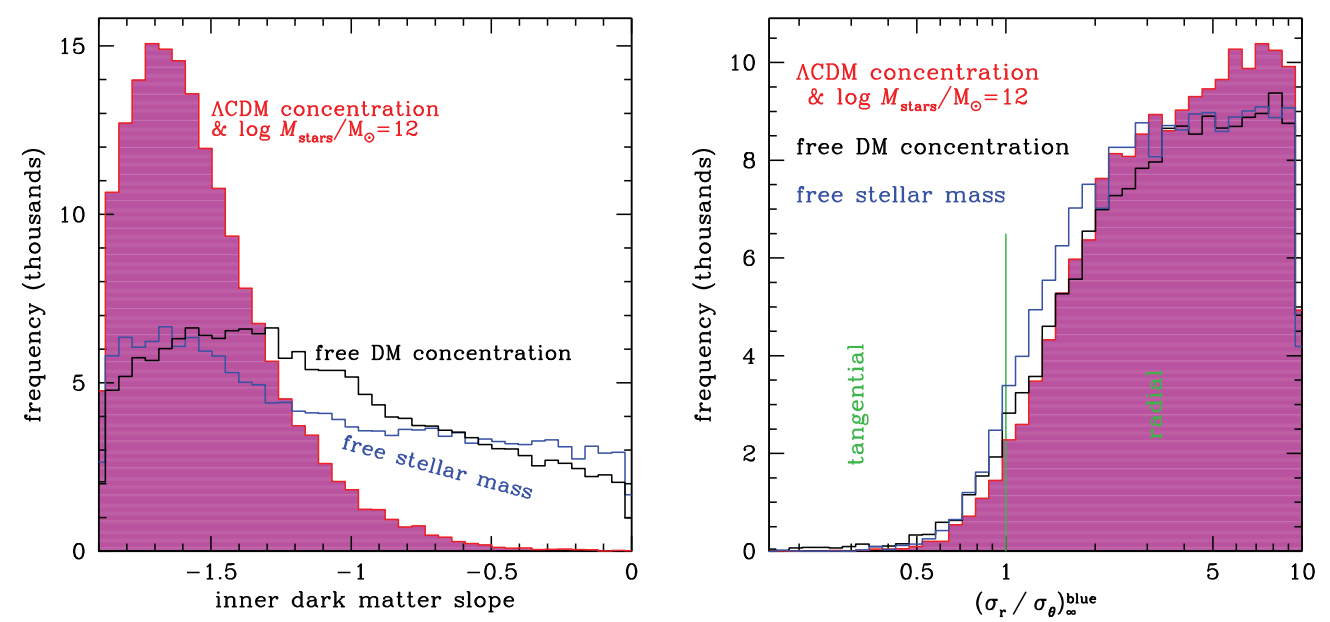

Figure 1. Effects of the choice of priors on the PDFs obtained with MAMPOSSt of the inner DM slope (left) and the outer VA of blue globular clusters (right) for M87 (traced by red and blue globular clusters). The pink shaded regions correspond to standard prior with $M_{\text {stars }} / L_{V}=8.4$ and the $\Lambda$ CDM DM concentration - virial mass relation, while the other two cases are for free stellar mass (blue, best fit corresponding to $M_{\text {stars }} / L_{V}=25$, which is abnormally high) or free DM concentration (black, best fit at $c=5$, a low value perhaps due to the Virgo cluster).

halos with Planck cosmology (Dutton \& Macciò 2014). Its stellar mass is fixed to $10^{12} M_{\odot}$ (yielding $M / L_{V}=8.4$ ). Our model also assumes Tiret et al. (2007) VA with isotropic inner velocities and a transition radius equal to the effective radius of the tracer.

The left panel of Figure 1 shows how $\gamma$ depends crucially on the priors of the model. While in our standard model $(\Lambda \mathrm{CDM}$ concentration and fixed stellar mass, pink shaded region) $\gamma=-1.75 \pm 0.25$, significantly cuspier than NFW, the models with either a free concentration (black histogram) or free stellar mass (blue) allow shallower slopes, including cores, although $\gamma<-1.3$ is again preferred. Interestingly, the outer VA of the blue GCs is always very radial in all three cases (right panel of Fig. 1). This analysis can be improved by including stellar velocity dispersions and the surrounding Virgo cluster.

\section{Fornax}

We extracted Walker et al. (2009)'s spectroscopic data for the Fornax dSph, only keeping stars with over $95 \%$ confidence of not being a foreground Milky Way star (according to the database of Walker et al. 2009), removing velocity outliers using the cuts from Lokas (2009), splitting between $Z$-poor and $Z$-rich components using a critical value for $W^{\prime} \equiv \Sigma \mathrm{Mg}^{\prime}$ of 0.5 (i.e., $Z$ rich corresponds to $W^{\prime}>0.5$ ). This yielded 722 and 1544 $Z$-rich and $Z$-poor stars, respectively. For both stellar components, we assume $n=0.71$ Sérsic profiles with $R_{e}=0.7 \mathrm{kpc}$ (Battaglia et al. 2006). We ran MAMPOSSt on a standard model with exponentially truncated DM (as found for tidally truncated subhalos by Kazantzidis et al. 2004) and free inner DM slope, Tiret et al. (2007) VA, and the $\Lambda$ CDM $c-M$ relation, allowing the total stellar mass to be free. Figure 2-left shows that the $Z$-rich population displays lower velocity dispersions at small projected radii $(R<0.3 \mathrm{kpc})$.

Figure 2-right shows that the inner DM slope is not well constrained, regardless of the priors on the DM concentration and stellar mass (despite very different best fits), because if the stellar mass is free, it dominates the DM within $2 R_{e}$ (with asymptotic $\log M_{\text {stars }}=8.2$ ). Finally, a robust finding is that the $Z$-rich outer VA is tangential. 

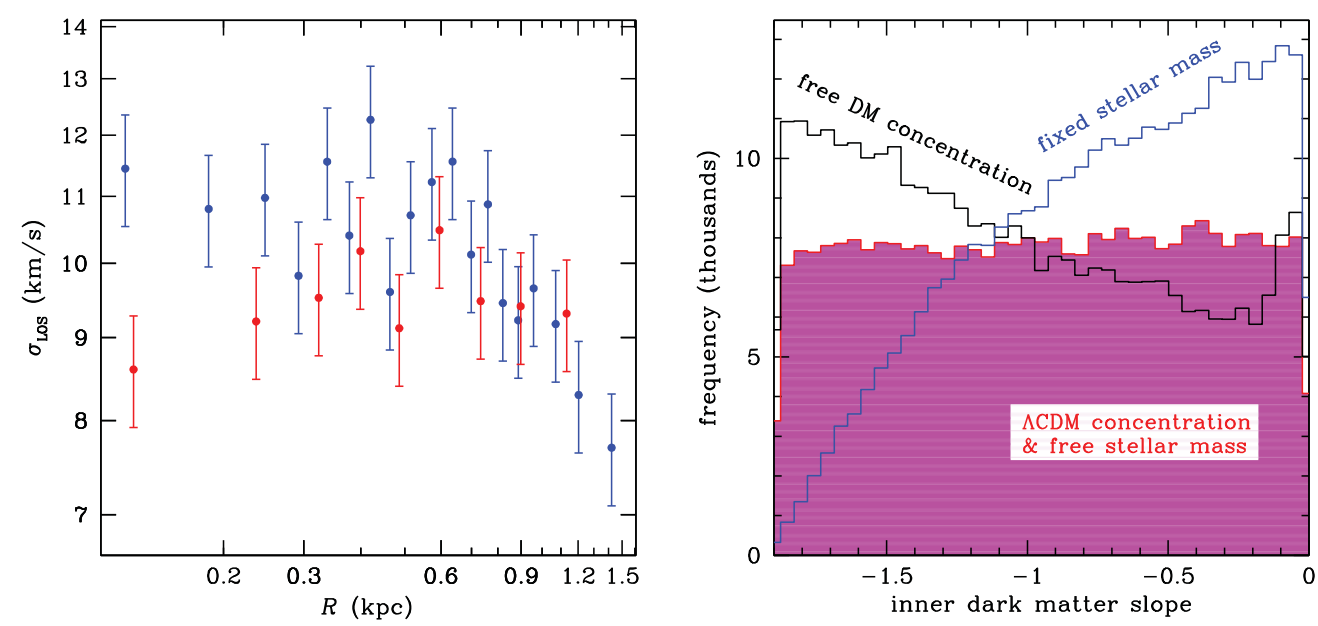

Figure 2. Analysis of the Fornax dSph. Left: Line-of-sight velocity dispersion profiles for the metal-rich (red) and metal-poor (blue) stars. Right: Effects of priors on the PDF of the inner DM slope of Fornax obtained with MAMPOSSt. The pink shaded region shows the standard prior with truncated outer DM, the $\Lambda$ CDM DM concentration - virial mass relation, and free stellar mass, while the other 2 cases are free DM concentration (black, best fit at $c=10$ ), or stellar mass fixed to $10^{7.95} M_{\odot}($ blue $)$.

\section{References}

Agnello, A., Evans, N. W., Romanowsky, A. J., \& Brodie, J. P., 2014, MNRAS, 442, 3299

Amorisco, N. C., Agnello, A., \& Evans, N. W., 2013, MNRAS, 429, L89

Battaglia, G. et al., 2006, A\&3A, 459, 423

Breddels, M. A., et al., 2013, MNRAS, 433, 3173

Courteau, S. et al., 2014, Reviews of Modern Physics, 86, 47

Dejonghe, H. \& Merritt, D., 1992, ApJ, 391, 531

Di Cintio, A., Brook, C. B., Macciò, A. V., et al., 2014, MNRAS, 437, 415

Dutton, A. A. \& Macciò, A. V., 2014, MNRAS, 441, 3359

Hearin, A. P. \& Watson, D. F., 2013, MNRAS, 435, 1313

Jardel, J. R. \& Gebhardt, K., 2012, ApJ, 746, 89

Kazantzidis, S., Mayer, L., Mastropietro, C., et al., 2004, ApJ, 608, 663

Łokas, E. L., 2009, MNRAS, 394, L102

Mamon, G. A., Biviano, A., \& Boué, G., 2013, MNRAS, 429, 3079

Murphy, J. D., Gebhardt, K., \& Adams, J. J., 2011, ApJ, 729, 129

Navarro, J. F. et al., 2004, MNRAS, 349, 1039

Pontzen, A. \& Governato, F., 2012, MNRAS, 421, 3464

Richardson, T. \& Fairbairn, M., 2013, MNRAS, 432, 3361

Richardson, T. \& Fairbairn, M., 2014, MNRAS, 441, 1584

Strader, J. et al., 2011, ApJS, 197, 33

Tiret, O., Combes, F., Angus, G. W., Famaey, B., \& Zhao, H. S., 2007, A\&\&A, 476, L1

Walker, M. G., Mateo, M., \& Olszewski, E. W., 2009, AJ, 137, 3100

Walker, M. G. \& Peñarrubia, J., 2011, ApJ, 742, 20

Wojtak, R., Łokas, E. L., Mamon, G. A., et al. 2009, MNRAS, 399, 812

Wojtak, R., Łokas, E. L., Mamon, G. A., et al., 2008, MNRAS, 388, 815

Wojtak, R. \& Mamon, G. A., 2013, MNRAS, 428, 2407

Wolf, J., Martinez, G. D., Bullock, J. S., et al., 2010, MNRAS, 406, 1220 